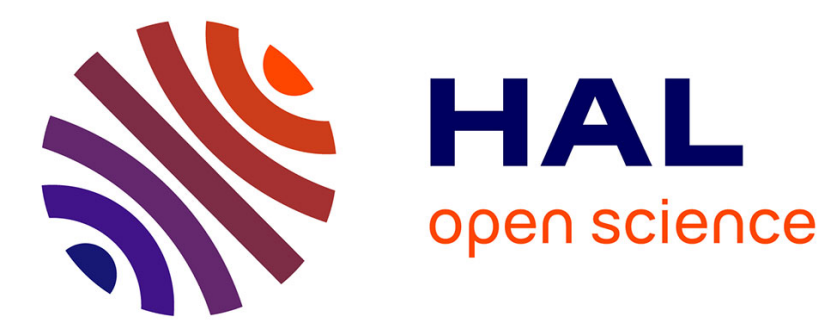

\title{
Syndrome d'apnées obstructives du sommeil : complications métaboliques
}

\author{
Elisabeth Frija-Orvoën
}

\section{To cite this version:}

Elisabeth Frija-Orvoën. Syndrome d'apnées obstructives du sommeil : complications métaboliques. Revue des Maladies Respiratoires, 2016, 10.1016/j.rmr.2015.11.014 . hal-01304949

\section{HAL Id: hal-01304949 \\ https://hal.sorbonne-universite.fr/hal-01304949}

Submitted on 20 Apr 2016

HAL is a multi-disciplinary open access archive for the deposit and dissemination of scientific research documents, whether they are published or not. The documents may come from teaching and research institutions in France or abroad, or from public or private research centers.
L'archive ouverte pluridisciplinaire HAL, est destinée au dépôt et à la diffusion de documents scientifiques de niveau recherche, publiés ou non, émanant des établissements d'enseignement et de recherche français ou étrangers, des laboratoires publics ou privés. 
RMR 140065

Syndrome d'apnées obstructives du sommeil : complications métaboliques

Obstructive sleep apnea: metabolic complications.

Frija-Orvoën Elisabeth

Université Pierre et Marie Curie

Titre court :

Auteur-correspondant : Frija-Orvoën Elisabeth

Université Pierre et Marie Curie

eofrija@gmail.com

Reçu le : 17.09.14

Accepté le : 16.11 .15

Aucun conflit à déclarer 


\section{Résumé}

Syndrome d'apnées obstructives du sommeil : complications métaboliques

Fortement lié à la présence d'une obésité, le SAOS est aussi un facteur de risque indépendant de dysfonctionnement du métabolisme du glucose allant de la simple intolérance au glucose au diabète de type 2. C'est également un facteur de risque de dyslipidémie, de syndrome métabolique, de stéatose hépatique non alcoolique. Les mécanismes de constitution de ces pathologies associées restent à préciser, l'hypoxie intermittente en étant sans doute une des clés. La place du traitement du SAOS dans leur prise en charge reste à préciser.

Mots clés : SAOS, diabète 2 , dyslipidémie, stéatose hépatique, PPC 
Summary

\section{Obstructive sleep apnea: metabolic complications.}

Strongly linked to the presence of obesity, OSA is an independent risk factor for abnormalities of glucose metabolism ranging from simple impaired glucose tolerance to type 2 diabetes. It is also a risk factor for dyslipidemia, metabolic syndrome and non-alcoholic fatty liver disease NAFLD. The pathological mechanisms underlying these associations remain to be precisely discovered, but intermittent hypoxia is probably one of the major factors.

The place of OSA treatment in the management of metabolic conditions remains unclear.

Keywords: OSA, 2 Diabetes, Dyslipidemia, Fatty Liver, PPC 
Le syndrome d'apnées obstructives du sommeil (SAOS) concerne $4 \%$ des hommes et $2 \%$ des femmes en population générale et sa prévalence ne cesse d'augmenter. II est caractérisé par un dysfonctionnement des voies aériennes supérieures conduisant à la succession au cours de la nuit d'arrêts complets (apnées) et/ou de diminutions significatives du débit aérien (hypopnées). Cependant le SAOS ne se résume pas à des problèmes mécaniques. C'est aussi un facteur de risque de développement de pathologies cardiovasculaires au premier rang desquels l'hypertension artérielle, de perturbation du métabolisme glucidique, de perturbation du métabolisme lipidique, de syndrome métabolique. Les liens avec les pathologies cardiovasculaires ayant été traités dans un autre article de la revue, nous nous attacherons ici à l'obésité et aux différents aspects métaboliques

\section{Obésité et SAOS}

L'obésité, définie par un indice de masse corporelle (IMC) supérieur à $30 \mathrm{~kg} / \mathrm{m}^{2}$, est un facteur majeur de développement d'un SAOS comme le montrent les études épidémiologiques.

Dans la Sleep Heart Health Study, la proportion de patients obèses est de $61 \%$ pour les index d'apnées-hypopnées (IAH) > 30/h (1). Dans l'étude publiée par Young en 2002 sur cette même cohorte, la proportion de SAOS est de $18 \%$ pour l'ensemble de la population avec $12 \%$ de patients pour un $I M C<25 \mathrm{~kg} / \mathrm{m}^{2}$ vs $32 \%$ pour un IMC $>30 \mathrm{~kg} / \mathrm{m}^{2}$ (2). Dans la cohorte de Cleveland suivie sur 5 ans, la survenue d'un SAOS est de $22 \%$ lorsque l'IMC 5 ans auparavant était $>31 \mathrm{~kg} / \mathrm{m}^{2}$ alors qu'il n'est que de $3,3 \%$ si l'IMC était $<24 \mathrm{~kg} / \mathrm{m}^{2}(3)$.

Dans l'étude longitudinale de Peppard, une augmentation de $10 \%$ du poids prédit une augmentation de $32 \%$ de l'IAH et multiplie par six le risque de développer un SAOS (4), tandis que la perte de poids diminue l'IAH de $26 \%$ (4).

Le suivi longitudinal de la cohorte de la Sleep Heart Health Study confirme le lien entre la prise de poids et l'augmentation de l'IAH. Dans cette étude, 2968 sujets ont été évalués à 5 ans d'intervalle et la prise de poids s'accompagne d'une augmentation de l'IAH (5). 
A contrario, les importantes pertes de poids obtenues après chirurgie bariatrique s'accompagnent dans la majorité des cas d'une franche diminution voire d'une normalisation de l'IAH (6).

L'obésité en cause est l'obésité dite viscérale $(7,8)$.

\section{L'obésité viscérale est un facteur majeur de développement d'un SAOS.}

Les moyens de lutte contre l'obésité, dont la chirurgie bariatrique, diminuent notablement I'IAH.

\section{SAOS et obésité}

Cependant, l'interférence SAOS et obésité est vraisemblablement plus complexe que le simple effet des dépôts graisseux abdominaux et pharyngés. Parmi les hypothèses soutenant un effet propre du SAOS sur l'obésité, on peut envisager la qualité du sommeil. II apparaît en effet que la dette de sommeil et/ou un sommeil de mauvaise qualité, comme peuvent en avoir les patients atteints de SAOS, modifient l'appétit et la régulation de la consommation alimentaire au profit d'une alimentation plus riche en aliments caloriques $(9,10)$.

Peu d'études se sont intéressées aux effets du traitement par PPC sur l'obésité et les résultats sont contradictoires. Dans l'étude de Redenius, on observe même une prise de poids sous PPC (11).

Le traitement par PPC pourrait-il, indépendamment d'une éventuelle perte de poids, avoir un effet sur l'accumulation de graisse viscérale observée dans le SAOS ?

Dans l'étude de Chin, chez 22 patients obèses avec SAS sévères, après 6 mois de PPC, on observe une diminution de la quantité de graisse viscérale chez les sujets dont le poids est stable et ceux dont le poids a diminué (12).

Sharma, après 3 mois de PPC efficace vs 3 mois de PPC factice, retrouve une petite diminution de poids sous PPC efficace $(-0,37 \pm 2,65 \mathrm{~kg})$ vs une tendance à la prise de poids $(0,33 \pm 2,2 \mathrm{~kg})$ sous PPC factice ainsi que sous PPC efficace une diminution de la graisse viscérale (13).

Par contre, Sivam dans son étude randomisée comparant des patients sous PPC efficace (utilisation moyenne 4,6 h/nuit) vs des patients sous PPC factice (utilisation moyenne $3,4 \mathrm{~h} /$ nuit) ne retrouve pas après 8 semaines de traitement, de modification 
de la quantité de graisse viscérale quel que soit le groupe (14). Ces résultats rejoignent ceux des études observationnelles de Vgontzas et Munzer $(15,16)$. Plus récemment, Hoyos chez 65 sujets de sexe masculin, obèses, après 12 semaines de PPC soit efficace soit factice, ne met pas en évidence de diminution de la graisse viscérale. À noter cependant dans cette dernière étude des utilisations moyennes de la PPC faibles, inférieures à $4 \mathrm{~h} /$ nuit (17).

II semble donc que les modifications de la graisse viscérale ne puissent être obtenues par le simple traitement par PPC mais nécessitent une perte de poids associée. À notre connaissance, il n'y a pas à ce jour d'étude sur l'effet à long terme d'un traitement par PPC sur la graisse viscérale.

La dette de sommeil et/ou un sommeil de mauvaise qualité sont souvent associés à une alimentation plus riche en aliments caloriques et donc à une obésité.

La PPC en elle-même ne semble pas induire de diminution de la graisse viscérale.

\section{SAOS et régulation du glucose}

\section{a) Tolérance à l'insuline}

Plusieurs études ont retrouvé un lien entre SAOS et dérèglement du système glucidique. Ainsi dans l'étude de Punjabi, après ajustement sur l'IMC, les sujets avec un $\mathrm{IAH} \geq 5 / \mathrm{h}$ ont un risque relatif de présenter une intolérance au glucose de 2,15 (18).

Des résultats identiques sont retrouvés par Elmasry. Chez des sujets en surpoids ou obèses modérés, la présence d'un IAH > 5/h multiplie par 2,15 le risque d'intolérance au glucose. Dans son étude, une insulinorésistance est retrouvée chez les patients SAS, obèses et non obèses. La saturation minimale est un facteur prédictif de l'insulinémie à jeun (19).

Dans l'étude de Ip, 280 sujets consécutifs adressés pour suspicion de SAOS, sans diabète connu, ont été explorés par polysomnographie. 185 SAOS ont été diagnostiqués. Les SAOS présentent plus fréquemment une résistance à l'insuline. L'insulinorésistance est liée à l'IAH $(p=0,044)$ et à la saturation minimale $(p=0,022)$. Dans cette étude, l'obésité joue un rôle mais le SAOS a un effet indépendant (20). 
Le ronflement, symptôme très fréquent du SAOS est lui-même facteur de risque de développement d'un diabète comme le montre l'étude longitudinale d'Elmasry. Sur une cohorte de 2668 hommes suivis 10 ans, la prévalence du diabète est de 5,4\% chez les ronfleurs versus $2,4 \%$ chez les non ronfleurs. Après ajustement sur le poids, la différence de prévalence dans le développement d'un diabète persiste entre ronfleur et non ronfleurs, 13,5 vs $8,6 \%$ (21).

Dans l'étude de Chin, sur 2719 hommes âgés de 40 à 60 ans, non obèses, si la glycémie et l'insulinémie à jeun ne sont pas différentes entre ronfleurs et non ronfleurs, le ronflement est associé à la glycémie lors du test de tolérance au glucose (22).

Une deuxième étude de Punjabi retrouve aussi que la diminution à la sensibilité à l'insuline est d'autant plus importante que l'IAH est élevé tout facteur confondant éliminé. La diminution de la sensibilité à l'insuline est corrélée avec le niveau de désaturation moyen (23).

Comme le montre la revue publiée par Punjabi en 2009, la majorité des études conclue à un lien entre le SAOS et la résistance à l'insuline indépendamment de l'âge, du sexe, de l'origine ethnique et de l'IMC (24).

Le SAOS s'accompagne de perturbations de l'équilibre glycémique.

L'augmentation de l'IAH est un facteur de risque d'insulinorésistance, et la diminution de la sensibilité à l'insuline semble corrélée au niveau de désaturation moyen.

Le ronflement en lui-même serait un facteur de risque de diabète.

b) Diabète

Dans la cohorte de la Sleep Heart Health Study, 9,6\% des sujets présentaient un diabète (25).

West interroge 1682 diabétiques par questionnaires (56\% de réponses). Le questionnaire utilisé est le questionnaire de Berlin et les sujets sont classés en forte probabilité $(57 \%)$ ou faible probabilité $(39 \%)$ de SAOS. Après une analyse oxymétrique, les sujets avec un index de désaturation $>10 / \mathrm{h}$ sont contrôlés par enregistrement du sommeil. La prévalence du SAOS chez le diabétique est estimée dans cette étude à $23 \%$. Le diabète y apparaît comme un facteur indépendant de SAOS (26). 
Reischmuth sur une cohorte de 1387 patients explorés par polysomnographie et suivis pendant 4 ans, retrouve une prévalence de $14,7 \%$ de diabète si l'IAH est $>15 / h$ versus $2,8 \%$ si l'IAH $<5 / h$. Le risque relatif de développer un diabète de type 2 dans les 4 ans est de 1,62 si l'IAH initial est $>15 \mathrm{~h}$ par rapport à un IAH initial < 5/h, ceci après ajustement pour l'âge, le sexe et l'IMC ()27.

Mahmood dans une cohorte de 1008 patients explorés consécutivement retrouve $74 \%$ de patients atteints de SAOS $(\mathrm{IAH}>5 / \mathrm{h})$. La prévalence du diabète est de $30,1 \%$ en cas de SAOS versus $18,6 \%$ en l'absence de SAOS. Cependant après ajustement sur les facteurs confondants, dans cette étude le SAOS n'est pas associé de façon indépendante avec le diabète (28).

Dans l'étude de Meslier, 595 patients suspects de SAOS ont réalisé un enregistrement du sommeil et un test de tolérance au glucose. $30 \%$ des sujets apnéiques présentaient un diabète vs $14 \%$ chez des ronfleurs non apnéiques. $40 \%$ des diabètes observés étaient méconnus. Dans cette étude, I'IAH est lié à la glycémie post-prandiale et à la sensibilité à l'insuline indépendamment de l'âge et de l'IMC (29).

Foster, chez 306 diabétiques explorés par polysomnographie, d'âge moyen $61,3 \pm 6,5$ et comportant $60 \%$ de femmes, retrouve $86,6 \%$ SAOS (IAH $>5 / h$ ). $22,6 \%$ d'entre eux présentent un SAOS sévère. Le meilleur critère prédictif de SAOS est le tour de taille : une augmentation d'un $\mathrm{cm}$ de tour de taille multiplie par dix le risque relatif de présenter un SAOS (30).

Ces résultats sont comparables à ceux de Laaban dont l'étude de 303 patients diabétiques, comportant $48 \%$ de femmes, hospitalisés pour diabète déséquilibré retrouve $63 \%$ de SAOS dont $10 \%$ de SAOS sévères. II est intéressant de noter que si les patients SAOS sont plus ronfleurs, la symptomatologie classique de somnolence diurne excessive, fatigue et nycturie ne diffère pas significativement quel que soit le groupe, apnéiques ou non et conduit les auteurs à proposer, compte tenu de la prévalence élevée, une recherche systématique de SAOS chez les patients diabétiques de type 2 même en l'absence de symptomatologie évocatrice (31).

Le diabète est un facteur de risque indépendant de SAOS.

Certains auteurs ont proposé une recherche systématique de SAOS chez les patients diabétiques de type 2 même en l'absence de symptomatologie évocatrice. 
c) SAOS et équilibre glycémique

Aronhson explorant 60 patients diabétiques retrouve $75 \%$ des patients avec un $\mid A H>5 / h$. Le niveau d'HbA1c est d'autant plus élevé que le SAOS est sévère : $+1,49 \%$ pour un SAOS léger, $+1,93 \%$ pour un SAOS modéré et $+3,69 \%$ pour un SAOS sévère (32).

Laaban ne retrouve pas de différence sur l'HbA1c quelle que soit la sévérité du SAOS mais les patients sélectionnés avaient tous un diabète déséquilibré justifiant leur hospitalisation (31).

Dans la cohorte française des pays de la Loire, chez des patients explorés pour SAS et sans diabète connu, l'IAH est indépendamment associé à HbA1c. Le pourcentage de sujets avec une $\mathrm{HbA} 1 \mathrm{C}>6 \%$ est de $10,8 \%$ pour un $\mathrm{IAH}<5 / \mathrm{h}, 34,2 \%$ pour un $\mid A H>50 / h$. Dans cette étude, il est également mis en évidence un lien entre les indices de désaturation et le risque d'avoir une $\mathrm{HbA} 1 \mathrm{c}>6 \%$ (33).

Ce lien avec le niveau d'oxygénation nocturne et l'hémoglobine glyquée est retrouvé par d'autres auteurs.

Dans l'étude de Tamura, 330 sujets suspects de SAOS, non obèses sont divisés en trois groupes selon leur statut glycémique : tolérance au glucose normale (164), tolérance au glucose altérée (111) et diabète de type 2 (55). La saturation minimale est associée au niveau de l'HbA1c quel que soit le statut glycémique (34).

De même, Sphirer chez 30 patients non connus comme diabétiques ou prédiabétiques mais dont les $2 / 3$ ont une $\mathrm{HbA1c}>6 \%$, retrouve une plus grande proportion de SAOS si HbA1c plus élevée. II note une corrélation avec la désaturation exprimée en moyenne et en temps passé à une saturation < $90 \%$ (35). $\mathrm{Au}$ total, il existe de plus en plus d'arguments pour considérer qu'il existe un lien indépendant entre le SAOS et la résistance à l'insuline et/ou le diabète.

Le SAOS est un facteur de risque de diabète et le niveau d'hémoglobine glyquée est d'autant plus élevé que le SAOS est sévère.

Il existe un lien entre le SAOS et la résistance à l'insuline et/ou le diabète.

d) Effet de la PPC sur le contrôle glycémique

Si le SAOS est un facteur indépendant du dérèglement de l'équilibre glycémique, le traitement devrait avoir un effet sur le contrôle glycémique. 
Dans l'étude de Harsh, la mise sous PPC améliore la tolérance à l'insuline dès le début du traitement ( $2^{\mathrm{e}}$ jour), amélioration confirmée à 6 mois. L'effet est d'autant plus marqué que les sujets ne sont pas obèses (36).

Dans l'étude de Babu, la mise sous PPC de patients diabétiques et atteints de SAOS améliore significativement les glycémies post-prandiales de chaque repas. Les patients qui avaient une $\mathrm{HbA} 1 \mathrm{c}$ supérieure à $7 \%$ voient celle-ci s'améliorer de façon significative. Cette amélioration est corrélée à la durée d'utilisation de la $P P C>4$ h/nuit (37).

De même Dawson, utilisant la mesure du glucose interstitiel nocturne chez des patients diabétiques et apnéiques, met en évidence, après 41 jours sous PPC, une amélioration des valeurs nocturnes de glucose et moins de variabilité de celles-ci sous traitement. Les valeurs de I'HbA1c ne sont cependant pas modifiées de façon significative (38).

Weinstock dans une étude randomisée en double aveugle et cross over, PPC efficace vs PPC factice, durée de traitement 8 semaines, ne retrouve pas d'effet du traitement par PPC sur l'intolérance au glucose. Seuls les patients ayant les SAS les plus sévères ont une amélioration de la tolérance à l'insuline (39).

Les résultats sur les effets du traitement par PPC sur le métabolisme du glucose sont cependant contradictoires mais les méthodes d'appréciation du métabolisme du glucose diffèrent d'une étude à l'autre, les durées de traitement également, il manque souvent des indications sur la compliance au traitement comme le souligne Chaktoura dans une revue générale publiée en 2012 (40).

Deux méta-analyses publiées respectivement en 2011 et 2013 sur les effets de la PPC sur l'équilibre glycémique montrent bien qu'il est nécessaire de disposer de plus d'études randomisées et si possible sur une plus longue durée pour conclure définitivement sur ce sujet (41-42).

Les données sur les effets du traitement par PPC sur le métabolisme du glucose sont contradictoires et des études complémentaires restent nécessaires.

\section{Durée du sommeil et tolérance au glucose}


Enfin il semble exister un lien entre la durée du sommeil et la tolérance au glucose. La privation de sommeil (4 heures par nuit pendant 5 jours consécutifs) chez des sujets jeunes, normaux, entraîne une diminution de la tolérance au glucose de 40 \% (43). La comparaison des effets sur un groupe de sujets sains d'une restriction de sommeil de 2 jours ( $4 h$ ) avec deux nuits de durée prolongée (10 h) montre qu'après les deux nuits de restriction, la glycémie est plus élevée au réveil et l'insulinémie plus basse (44).

Des sujets jeunes dont le sommeil est perturbé par des stimuli auditifs et mécaniques développent après deux nuits perturbées une diminution de la sensibilité à l'insuline ainsi qu'une élévation du cortisol (45).

La métanalyse de Cappucio publiée en 2010 confirme que la qualité et la quantité de sommeil représentent un risque de développer un diabète que l'on considère les courtes durées de sommeil $(<5-6 h, R R: 1,24)$, les longues durées ( $>8-9 h$, $R R: 1,48)$, les difficultés à s'endormir $(R R: 1,57)$ ou la difficulté à rester endormi (RR : 1,84) (46).

Les sujets atteints de SAOS ont un sommeil perturbé et ceci pourrait être aussi un des facteurs contribuant à la plus grande fréquence du diabète dans cette population indépendamment de l'obésité.

Par ailleurs, la qualité du sommeil pourrait influencer l'équilibre du diabète. Dans une population de 161 diabétiques interrogés sur leur durée de sommeil, après exclusion des sujets ayant des douleurs chroniques, la durée du sommeil est corrélée à l'équilibre du diabète apprécié par l'HbA1c (47).

Fendri compare 13 sujets obèses diabétiques et atteints de SAOS avec 13 sujets diabétiques obèses sans SAS. Les glycémies diurnes ne diffèrent pas dans les deux groupes mais les glycémies nocturnes sont $38 \%$ plus élevées en cas de SAS, indépendamment de l'obésité (48).

La privation de sommeil semble diminuer la tolérance au glucose.

Tous les troubles du sommeil (durée insuffisante, durée excessive, difficultés à s'endormir et difficultés à rester endormi) sont des facteurs de risque de diabète et de déséquilibre d'un diabète existant.

Le sommeil est perturbé au cours des SAOS, ce qui pourrait constituer un facteur de risque de diabète. 
Dans une étude portant sur des sujets diabétiques, les glycémies nocturnes, mais non les diurnes, étaient plus élevées en cas de SAOS, indépendamment de l'obésité.

\section{SAOS et dyslipidémie}

Dans la cohorte de la Sleep Heart Health Study, après ajustement pour l'âge et I'IMC, on retrouve une association significative avec l'hypercholestérolémie chez l'homme et avec l'hypertriglycéridémie chez la femme. Le taux de high density liprotein cholestérol (HDL-C) diminue avec l'accroissement de l'IAH (49).

Borgel retrouve sur une série de 366 patients une corrélation entre l'IAH et le HDL-C après ajustement sur l'âge, le sexe, l'IMC et le diabète. Le traitement par PPC a un effet positif sur le HDL-C (50).

Dans l'étude de Chou qui rapporte les résultats de 275 patients en surpoids ou obèses classe 1, 236 sont porteurs d'un SAOS défini par un $I A H>5 / h$. La prévalence de l'hypercholestérolémie est de $61,1 \%$, celle de l' hypertryglicéridémie de $55,3 \%$ et celle de l'hyperuricémie de $25,8 \%$. Dans l'analyse de régression logistique, la désaturation est une donnée indépendante contribuant à l'hypercholestérolémie et l'hypertriglycéridémie (51).

Dans l'étude de Tamura sur 308 sujets, on observe $71 \%$ de dyslipidémies (34).

Dans l'étude de Trzepizur, le bilan lipidique réalisé sur sa cohorte de 2081 patients, explorés par polysomnographie ou polygraphie ventilatoire, retrouve, après ajustement des facteurs confondants, que l'index de désaturation est associé à un niveau de triglycérides plus élevé et HDL-C plus faible (52).

Un effet de la PPC sur la dyslipidémie associée au SAOS est rapporté par l'étude de Phillips. II s'intéresse à la triglycéridémie postprandiale, marqueur plus puissant du risque cardiovasculaire que les valeurs des triglycérides à jeun.

Dans cette étude randomisée en crossover, la PPC améliore les pics postpandriaux de triglycérides, le cholestérol total, le cholestérol non HDL (53).

Une élévation de l'IAH s'accompagne de perturbation des bilans lipidiques, avec hypercholestérolémie et hypertriglycéridémie.

La PPC améliore les pics postpandriaux de triglycérides, le cholestérol total et le cholestérol non HDL. 


\section{SAOS et syndrome métabolique}

a) Syndrome métabolique

La définition du syndrome métabolique a évolué au fil des années. Le travail de différents experts a abouti à plusieurs propositions de définition du syndrome, les plus récentes étant celle du NECP (national cholesterol education program) adult treatment panel III (NECP-ATPIII) (54) et de I'International Diabetes Federation (IDF) (55) (tableau 1).

Quelle que soit la définition retenue, les éléments constituants du syndrome métabolique sont l'obésité viscérale, l'hypertension artérielle, la dysrégulation lipidique et glucidique. La présence d'un syndrome métabolique multiplie le risque de pathologie coronarienne par 2, celui de diabète par 4 .

Le syndrome métabolique est aussi associé à un état prothrombotique et proinflammatoire, également facteurs de risque de pathologie coronaire et de diabète de type 2.

La prévalence du syndrome métabolique dans l'étude NHANES III conduite entre 1988 et 1994 sur 8814 participants (4 265 hommes et 4549 femmes) âgés de 20 à 69 ans, est de $24 \%$ chez les hommes et $23,7 \%$ chez les femmes. Elle augmente avec l'âge passant de 6,7\% dans la tranche d'âge $20-29$ ans à $43,5 \%$ dans la tranche d'âge 60-69 ans (56).

En France, avec la définition de NECP-ATP III, la prévalence est de $9 \%$ chez les hommes, $6 \%$ chez les femmes selon les résultats de l'étude DESIR publiés en 2003 (57).

b) SAOS et syndrome métabolique

Différentes études ont mis en évidence une association SAOS et syndrome métabolique. Dans l'étude de Coughlin, 61 sujets obèses porteurs d'un SAOS sont comparés à 43 sujets contrôles. Après ajustement pour l'âge, l'IMC, le tabac, la consommation d'alcool, le SAOS est associé avec la PA, le HDL-C, l'hyperinsulinémie, l'hypertriglycéridémie. Le risque d'avoir un syndrome métabolique est 9 fois plus élevé chez les sujets SAOS (58). 
Dans l'étude de Gruber, où 38 SAOS sont comparés à 41 sujets contrôles, le risque relatif d'avoir un syndrome métabolique est de 5,9 (59).

De même, Lam sur une population chinoise de 215 sujets, 150 hommes et 105 femmes, explorés par polysomnographie, met en évidence un lien entre SAOS (seuil $\mathrm{IAH}>5 / \mathrm{h}$ ) et syndrome métabolique avec un risque relatif de 5,3 (60).

Dans l'étude rétrospective de Parish sur 228 patients consécutifs dont 146 atteints de SAOS, la prévalence du syndrome métabolique est plus élevée chez les SAOS : $60 \%$ vs $40 \%(61)$.

Dans l'étude de Sasanabe sur une population japonaise constituée de 719 hommes et 100 femmes, dont le diagnostic de SAOS a été fait par polysomnographie, la comparaison sujets SAOS et sujets non SAOS retrouve un lien SAOS-syndrome métabolique chez les hommes, $49,5 \%$ vs $22 \%$ comme chez les femmes $32 \%$ vs $6,7 \%(62)$.

Dans l'étude de Drager, chez 158 sujets chez lesquels a été découvert récemment un syndrome métabolique (critères NECPIII), l'exploration systématique du sommeil retrouve un $\mathrm{IAH}>15 / \mathrm{h}$ chez $60,5 \%$ des patients. Le SAOS est sévère chez $38 \%$ des sujets explorés. L'analyse des données met en évidence une association indépendante du SAOS avec deux critères du syndrome métabolique, les triglycérides et la glycémie à jeun, ainsi qu'avec le rapport cholestérol total-HDL cholestérol, l'uricémie, la protéine C-réactive. Parmi les paramètres du SAOS, ce sont I'IAH et la saturation minimale qui sont les variables associées aux paramètres biologiques ci-dessus (63).

Plus récemment, Lin sur une population de 154 sujets non obèses, sans augmentation de la graisse viscérale $\left(\mathrm{IMC}<25 \mathrm{~kg} / \mathrm{m}^{2}\right.$, périmètre abdominal $<90 \mathrm{~cm}$ chez les hommes et $80 \mathrm{~cm}$ chez les femmes) compare les sujets sans SAOS $(n=45)$ et les sujets avec SAOS. Ces derniers ont une pression systolique, un chiffre de triglycérides, une insulinémie plus élevés. La prévalence de l'hypertension artérielle, d'une dyslipidémie et d'au moins deux anomalies du syndrome métabolique est significativement plus élevée chez les sujets SAOS. L'IAH se révèle être un prédicteur indépendant de l'augmentation des triglycérides et de la résistance à insuline. La saturation minimale est inversement corrélée à la pression systolique (64).

c) Effet de la PPC sur le syndrome métabolique 
Il y a peu de données sur l'effet de la PPC sur le syndrome métabolique. L'étude randomisée, en cross-over de Coughlin sur 34 hommes testant une PPC efficace vs une PPS factice ne montre pas d'efficacité sur le syndrome métabolique mais une amélioration de la pression artérielle (65).

Plus récemment, Sharma dans une étude randomisée, double aveugle, avec crossover compare 3 mois de PPC efficace vs 3 mois de PPC factice. Dans sa population, un syndrome métabolique (selon les critères NECP-ATPIII) était présent chez $87 \%$ des patients. Sous PPC efficace, on observe une amélioration des paramètres $d u$ syndrome métabolique, et pour 11 patients ( $13 \%$ de l'effectif total) une disparition complète (66).

A contrario, De même, Hoyos ne retrouve pas d'effet sur le syndrome métabolique après 12 semaines de PPC (66).

Les données actuelles de la littérature sont encore insuffisantes pour conclure sur l'effet de la PPC sur le syndrome métabolique dans le SAOS.

Par ailleurs, la durée du sommeil pourrait aussi être un facteur à prendre en compte dans le lien SAOS-syndrome métabolique. Plusieurs études rassemblées dans une méta-analyse par Bo retrouvent en effet un effet potentiel du manque de sommeil sur la constitution d'un syndrome métabolique (67).

Le syndrome métabolique, caractérisé par une obésité viscérale, une hypertension artérielle, une dysrégulation lipidique et glucidique, multiplie le risque de pathologie coronarienne par 2 et celui de diabète par 4.

Le SAOS est un facteur de risque de syndrome métabolique.

L'IAH et la saturation minimale semblent être les variables associées avec la survenue d'un syndrome métabolique.

Le manque de sommeil pourrait être aussi un facteur de risque de syndrome métabolique.

L'effet de la PPC sur le syndrome métabolique reste à établir.

\section{SAOS et foie}

La stéatose hépatique non alcoolique est une pathologie fréquente allant de la simple infiltration graisseuse à la cirrhose. La résistance à l'insuline et 
l'hyperlipidémie sont des inducteurs de dépôts d'acides gras au niveau hépatique. Le SAOS qui favorise la résistance à l'insuline et la dyslipidémie pourrait de ce fait participer au développement de l'hépatopathie non alcoolique. Par ailleurs le SAOS est très lié à l'obésité elle-même également facteur de développement de stéatose hépatique non alcoolique.

Tanné, en 2005, sur une population de 163 sujets SAOS, retrouve une élévation des enzymes hépatiques chez $20 \%$ d'entre eux. II existe une association élévation des enzymes-IMC-SAS sévère. Huit sujets avec SAOS sévère (IAH > 50/h) ont eu une biopsie hépatique. Le pourcentage de stéatose lobulaire et de fibrose est corrélé avec l'IAH et le temps passé à une saturation inférieure à $90 \%$ (68).

Dans l'étude de Turkay, 106 sujets sont explorés à la fois par polysomnographie et sur le plan hépatique (dosages biologiques et échographie). 71 sujets présentaient des signes échographiques de stéatose hépatique. Comparés à ceux sans stéatose, ils étaient plus gros $(33,1$ vs $29,2, p=0,02)$ et présentaient plus de résistance à l'insuline. Après analyse multivariée, l'IAH, l'index de désaturation, la saturation minimale et le temps passé à une saturation inférieure à $90 \%$ s'avèrent des facteurs indépendants prédictifs de stéatose hépatique après prise en compte de l'IMC, l'âge, la résistance à l'insuline (69).

Dans l'étude de Mishra, 101 sujets vus avant chirurgie bariatrique ont eu un bilan hépatique. L'élévation des transaminases est corrélée aux marqueurs de l'hypoxie. Chez les 20 sujets ayant eu une biopsie hépatique, le SAOS est associé à la présence d'une hépatite stéatosique non alcoolique ainsi qu'à la résistance à l'insuline (70).

De même, l'étude d'Aron-Wisnewsky où 101 patients ont eu une chirurgie bariatrique et une biopsie de foie en peropératoire, confirme le rôle de l'hypoxie dans le développement des anomalies hépatiques. Les lésions de stéatose et de fibrose sont plus sévères si l'index de désaturation est plus élevé (71).

Une méta-analyse récente s'est intéressée aux travaux publiés sur ce sujet. 18 études transversales correspondant à l'inclusion de 2183 sujets, ont pu être analysées. Elle confirme un risque relatif plus élevé de stéatose hépatique non alcoolique et de fibrose en cas de SAOS (risques relatifs 2,16 et 2,30) (72).

En terme de suivi des anomalies hépatiques sous PPC, l'étude de Chin chez 40 sujets obèses et porteurs d'un SAOS sans atteinte hépatique ni consommation d'alcool qui présentaient une élévation des transaminases à l'état basal montre une 
diminution de ces valeurs après une nuit sous PPC, diminution persistante après 1 et 6 mois de traitement (73).

A contrario, Kholer comparant des sujets traités soit par PPC efficace soit par PPC factice ne retrouve pas d'effet sur les transaminases après 4 semaines de traitement (74).

La résistance à l'insuline et l'hyperlipidémie sont des inducteurs de dépôts d'acides gras dans le foie, avec apparition d'une stéatose hépatique.

Le SAOS, facteur de risque d'insulinorésistance et d'hyperlipidémie, pourrait donc également favoriser une stéatose hépatique.

La stéatose hépatique est également favorisée par l'obésité, fréquente au cours des SAOS.

L'IAH, l'index de désaturation, la saturation minimale et le temps passé à une saturation inférieure à $90 \%$ sont des facteurs indépendants prédictifs de stéatose hépatique.

L'effet de la PPC sur les anomalies hépatiques reste à déterminer.

\section{SAOS et marqueurs biologiques de l'inflammation}

La présence d'un SAOS induit deux phénomènes majeurs, une activation sympathique en rapport avec les éveils répétés, activation sympathique ayant des conséquences sur le système cardiovasculaire, et des épisodes d'hypoxie intermittente, tous événements aboutissant à un stress oxydatif. Les conséquences de ces phénomènes sont une réaction inflammatoire de bas grade dont il pourrait être intéressant de rechercher les stigmates chez les patients atteints de SAOS.

Différents marqueurs potentiels de cette inflammation comme la protéine C-réactive, le TNFa, les interleukines 6 et 8, l'adiponectine ont été recherchés avec des résultats variables.

Plusieurs études retrouvent des valeurs augmentées de protéine C-réactive. Ces valeurs élevées sont corrélées avec I'IAH, $(63,75,76)$ et ce indépendamment de l'obésité (77).

Dans une méta-analyse, Guo étudie les effets de la PPC sur la protéine C-réactive et conclut à une efficacité à partir de 3 mois de PPC si elle est utilisée $>4 \mathrm{~h} /$ nuit, 
efficacité qui se majore après 6 mois de traitement, ce qui pose le problème de la durée minimale de traitement pour obtenir un effet positif sur ce marqueur de l'inflammation (78).

Concernant le TNFa, les résultats sont variables soit augmentés en cas de SAOS (8,79-82) soit non modifiés (83). L'effet de 3 mois de PPC efficace semble positif sur un récepteur du TNF a $(84)$.

D'autres molécules sont diminuées comme l'adiponectine (85-87). Dans l'étude de Kholer sur l'effet de la PPC, 4 semaines de traitement ne modifient pas de façon significative le niveau d'adiponectine (88).

II ne se dégage pas au vu des résultats des études actuelles de profil spécifique concernant les marqueurs de l'inflammation et le SAOS.

La relation entre SAOS et marqueurs biologiques de l'inflammation reste encore mal précisée, tout comme les effets de la PPC (qui ne semble induire des modifications que si elle est prolongée).

En conclusion, le SAOS est lié à différents aspects de dysrégulation métabolique et ce indépendamment du facteur confondant majeur qu'est l'obésité. Les mécanismes en restent incomplètement élucidés mais l'hypoxie intermittente en est sans doute un élément essentiel. En cas de découverte d'un SAOS, il apparait licite de rechercher s'il existe une pathologie métabolique associée. A contrario, devant un diabète de type 2 ou une stéatose hépatique non alcoolique, il importe penser à rechercher un SAOS.

La place des traitements mécaniques du SAOS dans la prise en charge de ces pathologies métaboliques demanderait à être précisée.

\section{Points forts}

Le syndrome d'apnées obstructives du sommeil concerne $4 \%$ des hommes et $2 \%$ des femmes en population générale.

Le SAOS se caractérise par des apnées et/ou des hypopnées nocturnes.

Il s'associe souvent à une obésité.

Il induit plusieurs facteurs de risque cardiovasculaire : hypertension artérielle, perturbation du métabolisme glucidique, lipidique, et syndrome métabolique.

Ces facteurs de risque apparaissent indépendamment de l'obésité. 
Un diabète de type 2 ou une stéatose hépatique non alcoolique doivent faire rechercher un SAOS. 


\section{Questions}

\section{A Répondre par vrai ou faux}

1. Le seuil de l'obésité est un indice de masse corporelle supérieur à $30 \mathrm{~kg} / \mathrm{m}^{2}$.

2. L'obésité est un facteur de risque majeur de SAOS.

3. La chirurgie bariatrique permet de diminuer l'IAH chez les sujets obèses.

4. Un sommeil de mauvaise qualité s'accompagne d'une diminution des apports lipidiques alimentaires.

5. La PPC est efficace dans les SAOS, mais elle ne contribue pas à la diminution de la graisse viscérale.

6. Le seuil d'apparition des dérèglements du système glucidique au cours des SAOS est un $\mathrm{IAH} \geq 10 / \mathrm{h}$.

7. Le lien entre SAOS et résistance à l'insuline est plus marqué chez la femme.

8. Le diabète est un facteur de risque indépendant de SAOS.

9. II faut systématiquement rechercher un SAOS chez le diabétique de type 1.

10. La concentration d'hémoglobine glyquée est d'autant plus élevée qu'un SAOS est sévère.

11. II n'existe pas de lien entre les indices de désaturation nocturnes et la concentration plasmatique d'hémoglobine glyquée.

12. II existe un lien indépendant entre le SAOS et la résistance à l'insuline, mais non avec le diabète.

13. Il est clairement établi que la PPC améliore la tolérance à l'insuline dès le début du traitement.

14. Une durée excessive de sommeil est un facteur de risque de diabète.

15. Au cours des SAOS, les glycémies nocturnes sont plus élevées, ainsi que les glycémies diurnes, mais à un moindre degré.

16. La PPC améliore les pics postpandriaux de triglycérides, le cholestérol total et le cholestérol non HDL.

17. L'élévation de I'IAH est un prédicteur indépendant de l'augmentation des triglycérides plasmatiques.

18. Le SAOS est un facteur de risque de stéatose hépatique, vraisemblablement via une insulinorésistance et une hyperlipidémie.

19. Le SAOS, qui détermine une activation sympathique et des épisodes d'hypoxie intermittente, induit un stress oxydatif. 
20. Une PPC de plus de $4 \mathrm{~h} /$ nuit permet de diminuer la concentration de la protéine Créactive à partir de 3 mois de traitement.

\section{Réponses}

\section{A Vrai ou faux}

1. Vrai, 2.vrai, 3.vrai, 4.faux, 5.vrai, 6.faux, 7.faux, 8.vrai, 9.faux, 10.vrai, 11.faux, 12.faux, 13.faux, 14.vrai, 15.faux, 16.vrai, 17.vrai, , 18.vrai,19.vrai, 20.vrai 


\section{Références}

1 Nieto FJ, Young TB, Lind BK, Shahar E, Samet JM, Redline S et al. Association of sleep- disordered breathing, sleep apnea and hypertension in a large communitybased study: Sleep Heart Health Study. JAMA 2000; 283:1029-1036

Young T, Peppard PE, Gottlieb DJ. Epidemiology of obstructive sleep apnea: a population health perspective. Am J Respir Crit Care Med 2002; 165:1217-39

3 Tishler PV, Larkin EK, Schluchter MD, Redline S. Incidence of sleep-disordered breathing in an urban population: the relative importance of risks factors in the development of sleep-disordered breathing. JAMA 2003; 289:2230-7

4 Peppard PE, Young T, Palta M, Dempsey J, Skatrud J. Longitudinal study of moderate weight change and sleep-disordered breathing. JAMA 2000; 284:3015-21 Newman AB, Foster G, Givelber R, Nieto FJ, Redline S, Young T. Progression and regression of sleep-disordered breathing with changes in weight. Arch Inter Med 2005; 165:2408-2413

6 Greenburg DL, Lettieri CJ, Eliasson AH. Effects of surgical weight loss on measures of obstructive sleep apnea : a meta-analysis. The American Journal of Medicine 2009; 122:535-542

7 Shinohara E, Kihara S, Yamoshita S, Yamane M, Mshida M, Aras T, Kotani K, Kamatura K, Matsuzawa Y. Visceral accumulation fat as an important risk factor for obstructive sleep apnea syndrome in obese subjects. J Intern Med 1997;24:11-8

8 Vgontzas AN, Papanicolaou DA, Bixler EO, Hopper K, Lotsikas A, Lin HM, Kala A, Khronsos GP. Sleep apnea and daytime sleepiness and fatigue: relation to visceral obesity, insuline resistance and hypercytokinemia. J Clin Endocrinol Metab 2000; 85:1151-8

9 Spiegel K, Tasali E,Penev P, Van Cauter E. Brief communication sleep curtailment in healthy young men is associated with decreased leptin levels, elevated ghrelin levels and increased hunger and appetite. Ann Intern Med 2004; 141: 846-50

10 Marshall NS, Glozier N, Grunstein Rr. Is sleep duration relates to obesity? A critical review of the epidemiological evidence. Sleep Med Rev 2008; 12: 289-98

11 Redenius R, Murphy C, O'Neill E, Al-Hamwi M, Zallek SN. Does CPAP led to change in BMI? J Clin Sleep Med 2008;4: 205-209

12 Chin K, Shimizu K, Nakamura T, Narai N, Masuzaki H, Ogawa Y, Mishima M, najamura $T$, Nakao $K$, Ohi $M$. Changes in intra-abdominal visceral fat and serum 
leptine levels in patients with obstructive sleep apnea syndrome following nasal continuous positive airway pressure therapy. Circulation 1999; 100:706-7012

13 Sharma SK, Agrawal S, Damodoran D, Sreevinas V, Kadhivaran T, Laskshmy R, Jogra P, Kumar A. CPAP for metabolic syndrome in patients with obstructive sleep apnea. N Engl J Med 2011; 365:227-86

14 Sivam S, Phillips CL, Trenelle MI, Yee BJ, Liu PY, Wong KK, Grunstein RR. Effects of 8 weeks of continuous positive airway pressure on abdominal adiposity in obstructive sleep apnea. Eur Respir J 2012; 40:913-918

15 Vgontzas AN, Zoumakis E, Bixler EO, Lin HM, Collins B, Basta M, Pejovic S, Chrousos GP. Selective effects of CPAP on sleep apnoea-associated manifestations. Eur J Clin Invest 2008; 38:585-95

16 Münzer T, Hegglin A, Stannek T, Schoch $O$, Korte W, Büche D, Schmid C, Hürny C.Effects of long-term continuous positive airway pressure on body-composition and IGF1. Eur J Endociology 201; 162:695-704

17 Hoyos C, Lillick R, Yee BJ, Phillips CL, Grunstein RG, Liu PY. Cardiometabolic changes after continuous positive pressure for obstructive sleep apnea: a randomized sham-controlled study. Thorax 2012; 67:1081-1089

18 Punjabi NM, Sorkin JD, Katzel LI, Goldberg AP, Schwartz AR, Smith PL. Sleep disordered breathing and insuline resistance in middle-aged and overweight men. Am J Respir Crit Care Med 2002; 165:677-82

19 Elmasry A, Lindberg E, Berne C, Janson C, Gilason T, Tageldin A et al. Sleep disordered-breathing and glucose metabolism on hypertensive men: a population based study. J Int Med 2001; 249:153-61

20 Ip M SM, Lam B, NG M MT, Lam W K, Tsang K WT, Lam K SL. Obstructive sleep apnea is independently associated with insuline resistance. Am J Respir Crit Care Med 2002; 165:670-676

21 Elmasry A, Janson C, Lindberg E, Gilason T, Tageldin MA, Roman G. The role of habitual snoring and obesity in the development of diabetes: a 10 year follow-up study in a male population. J Int Med 2000; 248:13-20

22 Shin C, Kim JY, Kim JH, Lee SY, Shim JJ, In KH et al. Association of habitual snoring with glucose and insulin metabolism in non obese Korean adult men. Am J Respir Crit Care Med 2005; 171:287-291

23 Punjabi NM, Beamer B. Alterations in glucose disposal in sleep-disordered breathing. Am J Respir Crit Care Med 2009; 179:235-240 
24 Punjabi NM. Do sleep disorders and associated treatments impact glucose metabolism. Drugs 2009; 69 supp 2

25 Resnick HE, Redline S, Shahar E, Gilpin A, Newman A, Walter R et al. Diabetes and sleep disturbances. Findings from the Sleep Heart Health Study. Diabetes Care 2003; 26:702-709

26 West SD, Nicoll DJ, Stradling JR. Prevalence of obstructive sleep apnea in men with type 2 diabetes. Thorax 2006; 61:945-950

27 Reichmuth KJ, Austin D, Skatrud JB, Young T. Association of sleep apnea and type II diabetes. A population based study. Am J Respir Crit Care Med 2005; 172:15901595

28 Mahmood K, Akhter N, Eldeirawi K, Önal E, Christman JW, Carley DW, Herdegen JJ. Prevalence of type 2 diabetes in patients with obstructive sleep apnea in a multiethnic sample. J Clin, Sleep Med 2009; 5:215-221

29 Meslier N, Gagnadoux F, Giraud P, Person C, Ouksel H, Urban T, Racineux JL. Impaired glucose-insulin metabolism in males with obstructive sleep apnea syndrome. Eur Respir J 2003; 22:156-160

30 Foster GD, Sanders MH, Millman R, Zammit G, Borradaile KE, Newman AB, Wadden TA, Kelley D, Wing RR, Pi Sunyer FX, Darcey V, Kuna ST for the sleep AHEAD research group. Obstructive sleep apnea among obese patients with type 2 diabetes. Diabetes Care, 2009; 32:1017-1019

31 Laaban JP, Daenen S, Léger D, Pascal S, Bayon V, Slama G, Elgrably F. Prevalence and predictive factors of sleep apnea syndrome in type 2 diabetic patients. Diabetes 2\& metabolism 2009; 35:372-377

32 Aronsohn RS, Wihtmore H, Van Cauter E, Tasali E. Impact of untreated obstructive sleep apnea on glucose control in type 2 diabetes. Am J RespirCrit Care Med 2010; 181:507-513

33 Priou P, Le Vaillant M, Meslier N, Chollet S, Masson P, Humeau MP, Pigeanne T, Bizieux-Thaminy A, Goupil F, Gagnadoux F, the IRSR Sleppe cohort group Independant association between obstructive sleep apnea severity and glycated hemoglobin in adults without diabetes. Diabetes Care 2012; 35:1902-1906

34 Tamura A, Kawano Y, Watanabe T, Kadota J. Obstructive sleep apnea increases hemoglobin A1c levels regardless glucose tolerance status. Sleep Medicine 2012; 13 :1050-1055 
35 Shpirer I, Rapoport MJ, Stav D, Elizur A. Normal and elevated HbA1C levels correlate with severity of hypoxemia in patients with obstructive sleep apnea and decrease following CPAP treatment. Sleep Breath 2012; 16:461-466

36 Harsch I, Schachin S, Radespiel-Troger M, et al. Continuous positive airway pressure treatment rapidly improves insulin sensitivity in patients with obstructive sleep apnea syndrome. Am J Respir Crit Care Med 2004; 169:156-162

37 Babu AO, Herdegen JH, Fogefeld C, Shott S, Mezzoni T. Type 2 diabetes, glycemic control and continuous positive airway pressure in obstructive sleep apnea. Arch Int Med 2005; 165:447-452

38 Dawson AD, Abel SL, Loving RT, Sailey G, Shadan FF, Cronin JW, Kripje DF, Kline LE. CPAP therapy of obstructive sleep apnea in type 2 diabetics improves glycemic control during sleep. J Clin Sleep Med 2008; 4:538-542

39 Weinstock TG, Wang X, Prueschman M, Ismail-Bergi F, Aylor J, Babineau DC, Mehra $\mathrm{R}$, Redline S. A controlled trial CPAP therapy on metabolic control in individual with impaired glucose tolerance and sleep apnea. Sleep 2012; 35:617-625

40 Chaktoura M, Azar ST. Continuous positive airway pressure and type 2 diabetes mellitus. Diabetes \& metabolic syndrome : clinical research\&reviews 2012; 6:176-179

41 Hecht L, Mölher R, Meyer G. Effects of CPAP-respiration on markers of glucose metabolism in patients with obstructive sleep apnea syndrome : a systematic review and meta-analysis. Ger Med Sci 2011; 9:Doc20

42 Iftikhar IH, Khan MF, Das A, Magalang UJ. Meta-analysis: continuous positive airway pressure improves insulin resistance in patients with sleep apnea without diabetes. Ann Am Thorac Soc 2013; 10:115-120

43 Spiegel K, Leproult R, Van Cauter E.Impact of sleep debt on metabolic and endocrine function. Lancet 1999; 354:1435-9

44 Spiegel K, Knutson K, Leproult R, Tasali E, Cauter EV. Sleep loss: a novel risk factor for insulin resistance and type 2 diabetes. J Appl Physiol 2005; 99:2008-2019

45 Stamakis K, Punjabi NM. Effects of sleep fragmentation on glucose metabolism in normal subjects. Chest 2012; 137:95-101

46 Capuccio FP, Strazzulo P, D'Elia L, Miller MA. Quantity and quality of sleep and incidence of type 2 diabetes. A systematic review and meta-analysis. Diabetes Care $2010 ; 33: 414-420$ 
47 Knutson KL, Ryden AM, Mander BA, Van Cauter E. Role of sleep duration and quality in the risk and severity of type diabetes mellitus. Arch Inter Med 2006; 166:1768-1774

48 Fendri S, Rose D, Myambu S, Jeanne S, Lalau JD. Nocturnal hypoglycemia in type 2 diabetes with sleep apnea syndrome. Diabetes research and clinical practice 2011; 91:e21-e23

49 Punjabi NM, Ahmed MM, Polostky VY, Beamer BA, O'Donnell CP. Sleep disordered breathing, glucose intolerance and insulin resistance. Respir Physiol Neurobiol 2003; 16:167-78

50 Borgel J, Sanner BM, Bittlinsky A, Keskin F, Bartels NK, Buechner N, Huesing A, Rump LC, Mügge. A Obstructive sleep apnea and its therapy influence high-density lipoprotein cholesterol serum levels. Eur Respir J 2006; 27:121-127

51 Chou YT, Chuang LP, Li HS, Lin SW, Yang CT, Chen NH. Hyperlipidaemia in patients with sleep-related breathing disorders: prevalence and risk factors. Indian $\mathrm{J}$ Med Res, 2012;131:121-125

52 Trzepizur W, Le Vaillant M, Meslier N, Pigeanne T, Masson P, Humeau MP, BizieuxThaminy A, Goupil F Chollet, S Ducluzeau PH,Gagnadoux F. For the Institut de Recherche en Santé Respiratoire des Pays de la Loire (IRSR) Sleep Cohort Group. Independent association between nocturnal intermittent hypoxemia and metabolic dyslipidemia. Chest 2013; 143: 1584-1589

53 Phillips C, Yee BJ, Marshall S, Lui PY, Sullivan DR, Grunstein RR. Continuous positive airway pressure reduces postprandial lipidemia in obstructive sleep apnea $A$ randomized, placebo-controlled crossover trial. Am J Respir Crit Care Med 2011; 184:355-361

54 Executive summary of the third national cholesterol education program (NECP). Expert panel on detection, evaluation and treatment of high blood cholesterol in adults (Adult treatment panel III).JAMA 2001; 285:2486-2497

55 The IDF consensus worldwide definition of the metabolic syndrome in www.idf.org/webdata/docs/IDF_Metasyndrome_definition.pdf

56 Ford Es, Giles WH, Dietz WH. Prevalence of the metabolic syndrome among US adults: findings from the third National Health and Nutrition Examination Survey. JAMA 2002; 287:356-9 
57 Balkau B, Vernay M,Mahmdi L, Novak M, Vol S, Tichet $\mathrm{J}$ et al. The incidence and persistence of the NCEP (National Cholesterol Educatiion Program) metabolic syndrome. The French D.E.S.I.R. study. Diabetes Metab 2003; 29:526-32

58 Coughlin SR, Mawdsley L, Mugarza JA, Calverley P MA, Wilding J PH. Obstructive sleep apnea is independently associated with an increased risk of metabolic syndrome. European Heart Journal 2004; 25:735-741

59 Gruber A, Horwood F, Sithole J, Ali NJ, Idris I. Obstructive sleep apnea is independently associated with the metabolic syndrome but not insulin resistance state. Cardiovascular diabetology 2006; 5:22

60 Lam JC, Lam B, Lam CL, Fong D, Wang JK, Tse HF, Lam KS, Ip MS. Obstructive sleep apnea and the metabolic syndrome in community-based Chinese adults in Hong-Kong. Respir Med 2006; 100:980-987

61 Parish JM, Adam T, Facchiano L. Relation of metabolic syndrome and obstructive sleep apnea. J Clin Sleep Med 2007; 3:467-472

62 Sasanabe R, Banno K, Otake K, Hasegawa R, Usui K, Morita M, Chiomi T. Metabolic syndrome in japenese patients with obstructive sleep apnea syndrome. Hypertens Res 2006; 29:315-322

63 Drager LF, Lopes HF, Maki-Nunes C, Trombette IC, Toschi-Dias E, Alves MJ, Fraga RF, Jun JC, Negrão CE, Krieger EM, Polostky VY, Lorenzi-Filho G. The impact of obstructive sleep apnea on metabolic and inflammatory markers in consecutive patients with metabolic syndrome. PLoS One2010; 5 : e12065

64 Lin QC, Zhang XB, Chen GP, Huang DY, Din HB, Tang AZ. Obstructive sleep apnea syndrome is associated with some components of metabolic syndrome in non obese adults. Sleep Breath 2012; 16:571-578

65 Coughlin SR, Madwdsley L, Mugarza JA, Wilding JP, Calverley PM. Cardiovascular and metabolic effects of CPAP in obese men with OSA. Eur Respir J 2007; 29:720727

66 Hoyos CM, Sullivan DR, Liu PY. Effect of CPAP on the metabolic syndrome: a randomized sham-controlled study. Thorax 2013; 68:588-589

67 Bo X, Dan H, Zhang M, Xue J, Zhou D. Short sleep duration predicts risk of metabolic syndrome: a systematic review and meta-analysis. Sleep medicine Reviews 2013; 1 5 article in press 
68 Tanné F, Gagnadoux F, Chazoullières O, Fleury B, Wendrine D, Larnier E, Lebeau B, Poupon R, Serfaty L. Chronic liver injury during obstructive sleep apnea. Hepatology 2005; 41:1290-1296

69 Turkay C, Ozol D, Kasapoglu B, Kirbas I, Yildirim Z, Yigitoglu R Influence of obstructive sleep apnea on fatty liver disease: role of chronic intermittent hypoxia. Respir Care 2012; 57: 244-249

70 Mishra P, Nugent C, Afendy A, Bai C, Ahatia P, Afendy M,Fang Y, Elariny H, Goodman Z, Younossi ZM. Apnoeichypopnoeic episodes during obstructive sleep apnea are associated with histological nonalcoholic steatohepatitis. Liver Int 2008; 28:1080-1086

71 Aron-Wisnewsky J, Minville C, Tordjman J, Levy P, Bouillot JL, Basdevant A, Bodessa P, Clement K, Pepin JL. Chronic intermittent hypoxia is a major trigger for non -alcoholic fatty liver disease in morbid obese. J Hepatol 2012; 56:225-33

72 Musso G, Cassader M, Olivetti C Rosina F, Carbone G, Gambino R. Association of obstructive sleep apnea with the presence and severity of non-alcoholic fatty liver disease. A systematic review and meta-analysis. Obesity Reviews 2013; 14:417-431

73 Chin K, Nakamura T, Takahashi K, Sumi K, Ogawa Y, MasuzakiH, Muro S, hattori M, Niimi A, Chiba T, Nakao K, Mishima M, Ohi M, Nakamura T. Effects of obstructive sleep apnea syndrome on serum aminotransferase levels in obese patients. Am J Med 2003; 114:370-6

74 Kholer M, Pepperell JCT, Davies Jo, Stradling JR. Continuous positive airway pressure and liver enzymes in obstructive sleep apnea: data from a randomized controlled trial. Respiration 2009; 78:141-146

75 Shamsuzzaman SM, Winnicki M, Lanfranchini P, Wolk R, Karas T, Accurso V, Somers VK. Elevated C-reactive protein in patients with obstructive sleep apnea. Circulation 2002; 105:2462-2464

76 Bhushan B, Guleria R, Misra A, Pandrey RM, Luthra K, Vikram NK. Obstructive sleep apnea correlates with C-reactive protein in obese asian Indians. Nur Metab Cardiovasc dis 2009; 19:184-9

77 Guven SF, Turkkani MH, Ciftci B, Ciftci TU, Erdogan Y. The relationship between high-sensitivity C-reactive protein and the severity of sleep apnea syndrome. Sleep Breath 2012; 16:217-21 
78 Guo Y, Pan L, Ren D, Xie X. Impact of continuous positive airway pressure on Creactive protein in patients with obstructive sleep apnea: a meta-analysis. Sleep Breath 2013; 17:495-503

79 Minoguchi K, K, Yokoe T, Minoguchi H, Watabe Y, Yamamoto M, Aelachi M. Elevated production of tumor necrosis factor a by monocytes in patients with obstructive sleep apnea syndrome. Chest 2004; 126:1473-9

80 Cifti T, Korkturk O, Bukan N, Bilgihan A. The relations ship between serum cytokines levels with obesity and obstructive sleep apnea syndrome. Cytokines 2004; 28:87-91

81 Tamaki S, Yamauchi M, Fukuoka A, Makidonan K, Koyama N, Tomoda K, Yoshikawa M, Kimura $\mathrm{H}$. Production of inflammatory mediators by monocytes in patients with obstructive sleep apnea syndrome. Inter med 2009; 48:1255-1262

82 Steiropoulos P, Papanas N, Nerra E, Autoniadou M, Serasli E, Papoti S, Hatzizisi O, Kyriazis G, Tzouvelekis A, Maltezos E, Tsara V, Bouros D. Inflammatory markers in middle aged obese subjects: does obstructive sleep apnea syndrome play a role? .Mediators of inflammation 2010; 65:6753320

83 Hargens TA, Guill SG, Kaleth AS, Nickols-Richardson SM, Miller LE, Zedalis D, Gregg JM, Gwazdauskas F, Herbert WG. Insulin resistance and adipose-derived hormones in young men with untreated obstructive sleep apnea. Sleep Breath 2013; 17:403-409

84 Aria MA, Garcia-Rio F, Alonso-Fernandez A, Hernanz A, Hidalgo R, Martinez-Mateo V, Bartolomé S, Rodriguez-Pradial L. CPAP decrease plasma levels of soluble tumor necrosis factor a receptor 1 in obstructive sleep apnea...Eur Respir J 2008; 32:100915

85 Masserini B, Morpurgo PS, Donadio F, Baldessari C, Bossi R, Bech-Peccoz P, Orsi E. Reduced levels of adiponectine in sleep apnea syndrome.J Endocrinol Invest 2006; 29:700-5

86 Kanbay A, Kokturk O, Cifti TU, Tavil Y, Bukan N. Comparison of serum adiponectine and tumor necrosis factor a levels between patients with and without sleep apnea syndrome. Respiration 2008; 76:324-30

87 Nagakawa Y, Kishida K, Kihara S, Yoshida R, Funasahshi T, Shimomura I. Nocturnal falls of adiponectin levels in sleep apnea with abdominal obesity and impact of hypoxia-induced dysregulated adiponectin production in obese murine mesenteric adipose tissue. J Atheroscler Thromb 2011; 18:240-7 
88 Kohler M, Ayers L, Pepperell JCT, Packwood KL, Ferry B, Crosthwaite N, Craig S, Siccoli MM, Davies RJO, Stradling JR. Effects of continuous positive airway pressure on systemic inflammation in patients with moderate to severe obstructive sleep apnea : a randomized controlled trial. Thorax 2009; 64:67-73 
Tableau 1 : Définitions du syndrome métabolique

\begin{tabular}{|l|l|l|}
\hline Critères & NECP-ATPIII (2001) & IDF (2005) \\
\hline Glycémie $\mathrm{mg} / \mathrm{dl}$ & $\begin{array}{l}\geq 110 \text { ou diabète } \\
\text { connu }\end{array}$ & $\geq 100$ ou diabète connu \\
\hline $\begin{array}{l}\mathrm{HDL} \text { cholestérol } \\
\mathrm{mg} / \mathrm{dl}\end{array}$ & $<40(\mathrm{H}), 50(\mathrm{~F})$ & $<40(\mathrm{H}), 50(\mathrm{~F})$ \\
\hline $\begin{array}{l}\text { Triglycérides } \\
\mathrm{mg} / \mathrm{dl}\end{array}$ & $\geq 150$ & $\geq 150$ \\
\hline $\begin{array}{l}\text { Pression } \\
\text { artérielle } \mathrm{mmHg}\end{array}$ & $\begin{array}{l}\geq 130, \geq 85 \text { ou } \\
\text { traitement }\end{array}$ & $\geq 130, \geq 85$ ou traitement \\
\hline IMC $\mathrm{kg} / \mathrm{m}^{2}$ & $\begin{array}{l}\text { Périmètre abdominal } \\
\mathrm{cm}\end{array}$ & $\begin{array}{l}\text { Périmètre abdominal } \mathbf{c m} \\
\mathbf{9 4}(\mathrm{H}), \mathbf{8 0}(\mathbf{F})\end{array}$ \\
\hline $\begin{array}{l}\text { Obésité } \\
\text { viscérale }\end{array}$ & $>102(\mathrm{H}), 88(\mathrm{~F})$ & $($ Européens $)$ \\
\hline & 3 critères & Critère obligatoire +2 \\
\hline
\end{tabular}

Critère obligatoire : en gras. Pour IDF, le critère périmètre abdominal est variable selon l'ethnie. Les personnes avec une histoire de diabète et/ou d'hypertension artérielle sont classées comme ayant une glycémie et/ou une pression artérielle élevée. 